\author{
Series A
}

\author{
I. MATHEMATICA
}

499

\title{
BOUNDED ANALYTIC FUNCTIONS WITH LARGE CLUSTER SETS
}

A. J. LOHWATER and GEORGE PIRANIAN 
Copyright $(\mathcal{C} 1971$ by

Academia Scientiarum Fennica

Communicated 13 September 1971 by OLLI Lehto 
An analytic function $w=f(z)$ in the unit disk $D$ of the $z$-plane is called an inner function if $|f(z)|<1$ in $D$ and if $f(z)$ possesses radial limits of modulus 1 at almost all points of $|z|=1$. Such functions were studied extensively by O. Frostman [4] and W. Seidel [6]. In particular, at each point $P$ on $|z|=1$ the cluster set $C(f, P)$ of an inner function consists either of a single point of modulus 1 or else of the closed disk $|w| \leq 1$ (see [6, Theorem 6 and its corollary]).

Every bounded analytic function in $D$ has a representation

$$
f(z)=e^{i \alpha} B(z) \exp \left(\frac{1}{2 \pi} \int_{-\pi}^{z} \frac{e^{i t}+z}{e^{i t}-z} d \mu(t)\right),
$$

where $\alpha$ is a real constant, $B(z)$ is a Blaschke product extended over the zeros of $f(z)$, and $\mu(t)$ is a nonincreasing function (see [1, p. 40], for example). The function $\mu$ has a decomposition $\mu=\mu_{1}+\mu_{2}$, where $\mu_{1}$ is singular and $\mu_{2}$ is absolutely continuous. The inner functions are those bounded analytic functions for which $\mu_{2}$ is constant. Bounded analytic functions that have no zeros and for which $\mu_{1}$ is constant are called outer functions.

The following question arises naturally.: If $f(z)$ is analytic and bounded in $D$ and if at each point $P$ of $|z|=1$ the cluster set $C(f, P)$ consists either of a single point of modulus 1 or else of the closed disk $|w| \leq 1$, must $f(z)$ be an inner function? In case the answer is negative, do there exist outer functions with this property? Questions such as these have been raised by G. Csordás [2] and L. Rubel (private communication).

In our first theorem, we show that there exists a bounded analytic function whose cluster set $C(f, P)$ is the closed disk $|w| \leq 1$ for every $P$, but which has no radial limit of modulus 1 ; this answers the first question of the preceding paragraph. In Theorem 2, we show that an appropriate refinement of the simple construction in Theorem 1 leads to a function with the same property and with the additional feature that the new function has a finite Dirichlet integral. In Theorem 3, we show that there exist outer functions with both properties.

Theorem 1. There exists an analytic function $w=f(z)$ in $D$ such that $|f(z)|<1$ in $D$, such that for each point $P$ on $|z|=1$ the cluster set

G. Piranian acknowledges support from the National Science Foundation. 
$C(f, P)$ is the closed disk $|w| \leq 1$, and such that $f(z)$ does not have a radial limit of modulus 1 at any point $P$.

Proof. We shall construct a special domain $G$ in $|w|<1$, form the universal covering surface $G^{\infty}$ over $G$, and show that each conformal mapping of $D$ onto $G$ generates a function $f$ with the required properties.

We create a domain $G_{0}$ by deleting from the disk $|w|<1$ a curve $\sigma$ that spirals from the origin toward the circle $|w|=1$. From $G_{0}$, we obtain $G$ by the deletion of a denumerable set $E_{0}$ whose derived set consists of the curve $\sigma$ and the circle $|w|=1$; we subject $E_{0}$ to the additional requirement that if $\delta$ is any disk whose center lies on $\sigma$, then each component of $\delta \cap G_{0}$ contains points of $E_{0}$. Clearly, $G$ has infinite connectivity, and its boundary consists of the set $E_{0}$, the spiral $\sigma$, and the circle $|w|=1$.

Let $\varphi$ denote a conformal mapping of $D$ onto $G^{\infty}$, and let $f$ be the composition of $\varphi$ with the projection of $G^{\infty}$ onto $G$. Then $f$ is analytic in $D,|f(z)|<1$, and $f(z)$ assumes each value in $G$ infinitely often. From the general theory of inverse functions (see [1, Chapter 6], for example) it follows that each point of the set $E_{0}$ is the radial limit of $f(z)$ on a set that is dense on $|z|=1$. From this it follows in turn that for each point $P$ on $|z|=1$, the set $C(f, P)$ consists of the closed disk $|w| \leq 1$.

To show that no radial limit of $f$ has modulus 1 , we merely observe that no path on the Riemann surface $G^{\infty}$ converges to a single point on $|w|=1$.

In the statement of the following theorem, we call a point set a boundary path in $D$ if it consists of the values $z=g(s)$, where $g(s)$ denotes a continuous function $(0 \leq s<1)$ such that $|g(s)|<1$ for all $s$ and $\lim _{s \rightarrow 1}|g(s)|=1$.

THEOREM 2. There exists an analytic function $w=f(z)$ in $D$ such that

(i) $|f(z)|<1$ in $D$,

(ii) for each point $P$ on $|z|=1$, the cluster set $C(f, P)$ consists of the closed disk $|w| \leq 1$,

(iii) for each boundary path $\lambda$ in $D$, the cluster set $C_{i}(f, P)$ either contains no point of the circle $|w|=1$ or else consists of the entire closed disk $|w| \leq 1$

(iv) the Dirichlet integral of $f$ is finite,

(v) the set of Fatou values of $f$ has two-dimensional measure $\pi$.

Proof. Let $\gamma$ denote a path in $|w|<1$ that begins at $w_{1}=1 / 2$ and passes exactly once through each of the points $w_{2}, w_{3}, \ldots$, where

$$
w_{n}= \begin{cases}2^{-n} & (n \text { odd }), \\ 1-2^{-n} & (n \text { even })\end{cases}
$$




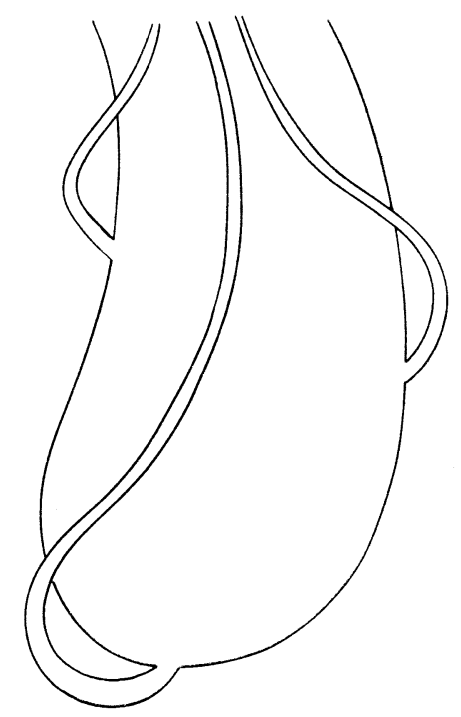

( $\gamma$ cannot be a boundary path). We may assume that for each $n$ the arc $\gamma_{n}$ from $w_{n}$ to $w_{n+1}$ is simple and rectifiable, and that no point of $|w|<1$ lies at a distance greater than $1 / n$ from $\gamma_{n}$. Let $\gamma$ have the representation $w=w(s)(0 \leq s<\infty)$, where $s$ denotes arc length on $\gamma$. For each $s$, let $D_{s}$ denote the disk $|w-w(s)|<1 / 8 e^{s}$; without loss of generality, we may assume that each disk $D_{s}$ lies at a positive distance from the circle $|w|=1$. Obviously, the disks $D_{s}$ determine a simply connected Riemann surface $G_{1}$ in the form of a ribbon that winds over the disk $|w|<1$. We divide the portion of the boundary of $G_{1}$ over the disk $|w|<1$ into arcs of diameter at most $1 / 2$, and along each of these arcs we attach to $G_{1}$ a Riemann surface that lies over $G_{1}$ (except for a short section near the attachment; see the figure) and follows its contortions indefinitely.

When the Riemann surface $G_{n}$ has been constructed, we divide the portion of its boundary over the disk $|w|<1$ into ares of diameter at most $2^{-n}$; along a portion of each of these arcs we attach to $G_{n}$ a Riemann surface that lies over the corresponding ribbon of $G_{n}$ (except for a short portion) and follows it indefinitely; and we denote the Riemann surface thus obtained by $G_{n+1}$. The continuation of the process yields a simply connected Riemann surface $G^{*}$, and each conformal mapping of $D$ onto $G^{*}$ determines a function $f$ with the first two of the properties listed in the theorem. To see that condition (iii) is also satisfied, we merely observe that if the projection of a path in $G^{*}$ has a limit point on the circle $|w|=1$, then the projection of path is dense on the unit disk.

To ensure that the functions $f$ associated with $G^{*}$ satisfy condition 
(iv), we need only make certain that the total area of the Riemann surfaces comprising $G^{*}$ is finite.

Finally, to make certain that almost all points in the disk $|w|<1$ are Fatou values of the functions $f$, it is simplest to abandon the rectifiability of the ares $\gamma_{n}$ that comprise the path $\gamma$ (naturally, this also requires modifications in the definition of the disks $D_{t}$ ). We can then choose the path $\gamma$ and the supporting Riemann surface $G_{1}$ so that the projection of the set $B_{1}$ of its accessible boundary points has measure at least $3 \pi / 4$. Whenever we attach a Riemann surface to $G_{1}$, we do it in such a way that the set of accessible boundary points of $G^{*}$ contains a subset of $B_{1}$ whose measure is at least $\pi / 2$. Similarly, we make certain that the projection of the set of accessible boundary points of the Riemann surface $G_{m}$ has measure at least $\left(1-2^{-m-1}\right) \pi$, and that a set of measure at least $\left(1-2^{-m}\right) \pi$ survives all later modifications. This completes the proof of Theorem 2.

In connection with condition (iv), we observe that if $f$ is a univalent map of the disk $D$ onto $G^{*}$, then at each point $e^{i \theta}$ the radial cluster set of $f$ consists either of an interior point of the disk $|w|<1$ or else of the closure of the disk. By a slight modification of the classical proof of a theorem of Fejér (see [3] or [5, Section 13]), we see that at each point $e^{i(}$, the sequence of partial sums of the Taylor series of $f$ either converges or has each point in $|w| \leq 1$ as a limit point.

TheOREm 3. There exists an outer function with the properties listed in Theorem 2.

Proof. In the proof of Theorem 2, we can obviously construct the Riemann surface $G^{*}$ so that the point $w=0$ is the projection of no interior point of $G^{*}$ and of no accessible boundary point of $G^{*}$. Each of the associated functions $f$ is then an outer function; for if in the decomposition $\mu=\mu_{1}+\mu_{2}$ of the function $\mu$ in (1) the component $\mu_{1}$ were not constant, there would be at least one point $t_{0}$ such that $\mu_{1}^{\prime}\left(t_{0}\right)=-\infty$, and at the point $e^{i t_{0}}$ the function $f$ would have the radial limit 0 .

Case Western Reserve University and

The University of Michigan 


\section{References}

[1] Collingwood, E. F., and Lohwater, A. J., The Theory of Cluster Sets, Cambridge University Press, Cambridge, 1966.

[2] Csondás, G., The Šilov boundary and a class of functions in $H^{\infty}$, Ph. 1). Dissertation, Case Western Reserve Univ., Cleveland, Ohio, 1969.

[3] Fejér, L., Über die Konvergenz der Potenzreihe an der Konvergenzgrenze in Fällen der konformen Abbildung auf die schlichte Ebene, Mathematische Abhandlungen, Hermann Amandus Schwarz zu seinem fünfzigjährigen Doktorjubiläum am 6. August 1914 gewidmet von Freunden und Schülern, pp. $42-53$, Berlin, 1914 .

[4] Frostman, O., Potentiel d'équilibre et capacité des ensembles avec quelques applications à la théorie des fonctions, Medd. Lunds Univ. Mat. Sem., Band 3, 1935, pp. 1-118.

[5] Landau, E., Darstellung und Begründung einiger neuerer Ergebnisse der Funktionentheorie, Chelsea Publ. Co., New York, 1946.

[6] Seidel, W., On the distribution of values of bounded analytic functions, Trans. Amer. Math. Soc. 36 (1934), $201-226$. 